\title{
3 Research Square \\ On The Functional Dynamics of Alveolar and Ductal Growth in The Adolescent Lung
}

Rex Moats ( $\square$ RMoats@chla.usc.edu )

Children's Hospital Los Angeles

\section{David Koos}

Children's Hospital Los Angeles

Wei Shi

Children's Hospital Los Angeles

Yang Tang

Children's Hospital Los Angeles

Harvey Pollack

Children's Hospital Los Angeles

Dnyanesh Tipre

Children's Hospital Los Angeles

Thien Khuu

Children's Hospital Los Angeles

Vlad Larionov

Children's Hospital Los Angeles

Clarence Wigfall

Children's Hospital Los Angeles

Merryn Tawhai

Children's Hospital of Los Angeles

\section{Scott Fraser}

University of Southern California https://orcid.org/0000-0002-5377-0223

\section{David Warburton}

Children's Hospital of Los Angeles

\section{Article}

Keywords: Alveolar, Adolescent Lung, 3D, adolescence

Posted Date: June 30th, 2021

DOI: https://doi.org/10.21203/rs.3.rs-632711/v1 
License: (c) (i) This work is licensed under a Creative Commons Attribution 4.0 International License. Read Full License 


\section{Abstract}

During adolescence, the lungs expand while alveoli increase greatly in number. A precise and deep understanding of the biological processes responsible for the many fold increase in alveoli during adolescence could form the foundation for novel treatments to reoptimize lung function after disease or injury. Herein we provide a unique insight into the alveolar growth in the mouse lung during first postnatal 4 weeks herein described as the adolescence phase. Using 3-Dimensionnal (3D) high-resolution large field of view optical imaging coupled with qualitative and quantitative analysis, we suggest the bulk of alveolarization during adolescent lung growth occurs while the surface to volume of the acinar volume remains constant. The most distal 10 or more bifurcations within the acinar saccular region, comprised of small ducts and alveoli, are of similar scale. We explored the connections between the growth of lung and it's structural and functional parameters, by comparing the correlations between alveolar size, surface area of gas exchange regions, breathing rate, metabolic rate, and oxygen demand across mammalian species which exhibit widely divergent body sizes.

\section{Introduction}

The rate of lung growth from early childhood through adolescence up to young adulthood is remarkable and this growth occurs while the child runs, jumps, and plays. Throughout the adolescent stage of growth, we report that the lung maintains a stable surface-to-volume ratio of the alveolar ducts and alveolar regions within the lung acini. Fundamental insights focused on the bulk alveolarization phase occurring during adolescence is relevant not only to the design of scalable repair, regeneration, and reconstruction of the lung1, 2,3,4 but also for the prevention and treatment of disease. Decreased alveolar gas diffusion caused by the failure to develop or similarly the loss of these surfaces results in diminished peak lung function. These key characteristics of adolescent lung development and maturation have hitherto remained quite opaque, largely due to difficulties of studying the structure and function of an organ that is both in constant motion and undergoing constant expansion thus limiting direct optical observations especially at the submicron scale reported herein.

The lung is composed of repeating thin-walled gas exchange units which expand and contract during the respiratory cycle. During adolescence, lungs in mammalian species grow many times in volume over widely differing body size scales (mouse, monkey, cow, human, horse, whale) while simultaneously increasing alveolar number many-fold. The mouse, from which we draw our latest inferences, is not an exception. During its postnatal bulk growth phase from day 7 to 28 , the alveoli increase almost linearly with lung volume ${ }^{5}$. We therefore infer that this is the period of lung development that is most likely to be accessible to intervention and thus the most important window to prevent disease. Furthermore, mimicking or reconstitution of this bulk adolescent growth process may be attractable target in the optimization of repair of the lung. For example, childhood interstitial lung disease (ChILD) comprises a group of rare lung diseases that can alter the structure and function of the lungs in babies and children as well as teens so that early intervention could have a profound effect on a patient's long-term outcomes. 
Many previous publications have described lung alveolar growth from the early saccular structures in the pre and perinatal stage of development and into early childhood based largely on histology and morphometric approaches. These books and articles have contributed greatly to our understanding of lung development and its functional maturation ${ }^{6,7,8}$. However, lung growth in postnatal 7-28 days age groups and late stage alveolarization ${ }^{8}$ has remained a relatively unexplored area, although it represents the period in which the lung expands to its greatest capacity, almost linearly with alveolar number.

In early development the region of the lung distal to the bronchoalveolar (BADJ) junction is termed the acinar saccule. The BADJ marks the sharp boundary transition between the airway conducting system where no gas exchange occurs, and the gas exchange surfaces that mediate the exchange of oxygen from the inspired air into the blood and the reverse exchange of carbon dioxide from the blood to the expired air. A clear example of a BADJ is labeled in Fig. 1 and the figure provides the reader with an understanding of the spatial dimensions of our study relative to the lung air exchange surface of an acinar region. Proximal to the BADJ the branching of the conducting airways is stereotypic, while distal to the BADJ the alveolar ducts continue to branch stereotypically at first but then branching becomes nonstereotypical and apparently random, resulting in a much more complex branching pattern ${ }^{9}$. Within one mouse lung, these distal branching networks contain more than 30,000 branch points and roughly twice as many segments, all of which have many associated alveoli ${ }^{10}$.

Air moves in out of the lung in a cycle of negative and positive pressures motivated by movements of the diaphragm and chest wall. Meanwhile, breathing continues as the lung grows in volume during adolescence. In the region of the mouse lung distal to the BADJ, termed the acinar saccule, airway components are comprised of ducts and their associated alveoli. The acinar gas exchange unit is a relatively large physiological unit containing 10,000 to 100,000 alveoli: $6.5-7.5 \times 10^{4}{\mu m^{2}}^{2}$ alveoli in an adult mouse and $12-80 \times 10^{4} \mu \mathrm{m}^{2} /$ alveoli in an adult human ${ }^{10,11,12}$. In turn the lung itself is composed of 1000's of these acinar units. We used 3D optical imaging at high resolution with a large field of view relative to the size of the functional lung gas exchange unit, the alveolus. We made repeated measurement during adolescent growth at postnatal 7-28 days in the mouse. We aimed to accurately follow the growth of the adolescent lung's surface to volume ratio and thus gain insight into factors determining the health and gas diffusion properties in the lung in adolescence and young adulthood.

Stereological analysis of serial 2-dimensional (2D) planar histological sections has been widely used to extract quantitative information about the 3D structure of the lung. Two recent rigorous 2D histology studies based on stereology followed the American Thoracic Society-based guidelines and defined sampling methodologies for the lung $5,10,13$ and produced similar results. These studies produced idealized 3D measurements of the average alveolus diameter and shape based on a series of static slices of $2 \mathrm{D}$ data samples within the lung. By direct measurement of the $2 \mathrm{D}$ histology, these studies showed that the average alveolar diameter and thus their volume stayed largely the same during adolescence. This was true while the alveoli multiplied linearly with the increase in cubic lung volume, although the authors did not choose to emphasize this conclusion ${ }^{5}$. Importantly, we emphasize that it follows from 
this that the significant lung volumetric increase during adolescence was primarily due to the formation of new alveoli, with an associated incremental increase in alveolar ducts, ductal length, ductal diameter and a concomitant increase in vascular tissue. An increase in ductal diameter of $40 \%$ effective doubles its cross-section area. Meanwhile the bulk of lung volume increase during adolescence comes from the formation of new alveoli.

Our 3D multi-scale mapping studies of adolescent mouse lung using a qualitative analysis led us to the same conclusion as the stereology-based papers, that alveolar size did not change significantly during adolescence. To verify our qualitative observations, we performed a quantitative analysis of the air exchange surface. Our regional study included the small alveolar ducts distal to the conducting airways, beginning slightly distal to the BADJ and extended to the pleural surface.

\section{Results}

Surface to volume ratio of alveoli. We precisely measured the surface to volume ratio of the exchange airway surfaces to accurately represent the changes occurring during the cubic growth of the acinar region. If in this late stage of alveolar development the erection of secondary septa is occurring as described for primary septa during early stages of alveolarization ${ }^{13,14}$ an increase to the surface to volume ratio is expected to occur, as bigger spaces are progressively subdivided by septal ridges into smaller spaces ${ }^{15}$. To test the similarity between these two stages of alveolarization we set out to measure the surface-to-volume relationship as accurately as possible in $3 \mathrm{D}$.

We used serial confocal tomography to image a developmental series of lungs prepared from transgenic mice that ubiquitously express a membrane targeted tdTomato fluorescent protein ${ }^{16}$. Left lungs from 21 mice aged from 7, 14, 21, and 28 postnatal days were included in this study. A typically prepared sample and imaging data is shown in Fig. 1 for one optical data set, clearly showing the exquisite level of complex detail afforded at an optical resolution of $0.42 \times 0.42 \mu \mathrm{m}$ in XY plane by $0.74 \mu \mathrm{m}$ in $\mathrm{Z}$ thickness. The BADJ, which lies at the most proximal portion of an acinar sac, can be appreciated along with the fine details of alveolar structure, together with large and small caliber veins, arteries and capillary networks. The characteristics of a typical data set are illustrated in Fig. 1 which provides a representative visualization of our data sets.

We created a customized segmentation pipeline focused on segmenting out the air exchange surface during adolescent lung growth. The method, based on the use of a Chan-Vese algorithm ${ }^{17}$, was chosen for the final analysis because it defined an airway surface closely modeling the actual surface. Rare manual adjustment was applied in some cases to avoid bridging across airway ducts caused by pores of Kohn or opaque imaging areas. From these 3D stacks, we segmented tissue and airway surfaces. Within a volume we analyzed which airway voxels were connected continuously with others, using a connected component analysis, thus separating the tissue components, locating and defining airway surfaces, and airway volumes from within the larger volume as regions of airways defined by their connectivity as show in Fig. 1. 
In order to focus on more distal airspaces, we focused on connected components located near the pleural surface, because there are fewer large ducts and non-gas exchange conductive airway in that location, while the pleura provides a point of distal orientation.

Figure 1 shows an extended volume of lung reconstructed from serial confocal tomography. Shown within the volume are several connected components, four neighboring distal components, and a larger connected component that includes the four distal ducts and a large branch of an artery that extends into the distal respiratory regions. The surface area to volume for each individually segmented airway connected component is plotted in Fig. 2a versus age.

The 96 connected components consisted of 8 separate volumes for 3 sets of samples, each at 4 different ages from early adolescence to young adulthood in mice. The average surface-to-volume ratio of alveoli on postnatal day 7, 14, 21 and 28 showed subtle differences: $0.089,0.092,0.109$, and $0.097 \mu^{-1}$, respectively. The mean difference of surface-to-volume ratio of only the 21 days old mice was statistically significantly difference from any of the other age groups (Fisher's LSD, $p<0.05$ ). The surfaceto-volume ratio slightly increased from postnatal day 7 to 21 and then decreased on day 28 . By plotting surface to volume ratio as a function of the volume analyzed, we observed that the surface-to-volume ratio is largely independent of the extent of the volume analyzed over a 1000-fold range (Fig. 2b).

The Y-intercept can be interpreted as representing the surface-to-volume ratio for the very smallest volumes which are individual alveoli. If we model acinar volumes as containing only alveolar spheres as represented by the $Y$-intercept, then the ratio of the surface-to-volume is simply $3 r$ ( $r$ = radius). Using this equation and the $Y$-intercept value of the average alveolus in the data sets as 0.102 , gives an average alveolar radius in mice of $29 \mu \mathrm{m}$.

Murine lung duct and alveoli analysis. We next focused our attention on the detailed analysis of one typical data set to more deeply determine the fine structure of the distal ductal branches and alveolar variation. We examined the microstructure of the alveoli and their associated ducts within a typical terminal alveolar region near the pleural surface. We concentrated on one segmented connected component from the eight selected for one 14 days old mouse data sets shown in Fig. 1 and shown individually in Fig. 3a.

After first optimizing the fitting parameters for our data we applied a mathematical tree algorithm using Amira 19.2 software (Thermo Fisher Scientific) to determine the topical branching pattern for the structure of our representative volume as shown in Fig. 3b. Larger ducts terminating at alveoli are shown as reddish brown circles. (Note: not to scale for visualization purpose the ducts being scaled down.) Fig. 3c contains all identified alveoli, shown as red dots and smaller ducts shown as tan branched segments. Figure $3 \mathrm{~d}$ is the same data with the ducts closer to their actual size, but still scaled down and color coded by their widths to aid visualization. Figure $3 a$ is at full scale for airway volume and the interstitial tissue is not present. This Amira- based analytical algorithm provided us with a branch point, a segment length, and a radius tangential to the axis of the segment. Ideally, the optimized mathematical 
representation of the terminal branch would be an alveolus, but due to the complex nature of the alveolar surface, the optimized algorithm still found branches or ductal segments depending on the surface contours within specific alveoli. To accurately assess the branched nature of terminal acinar regions, it was necessary to precisely define where a duct begins and ends. Thus, we wrote MATLAB scripts to make minor corrections to the output of the Amira tree algorithm, removing mathematical errors caused by the lack of consideration of biological scale.

Some individual alveoli were determined to be two or three mathematical endpoints of the tree if their surface was not smooth as previously noted ${ }^{18}$. We also observed that the occasional ductal segment would be very short because the algorithm added a short branch in relatively broad ductal regions where 3-6 alveoli were obtained to one location. We customized the tree-algorithm analysis to correct both for the very short ductal segments at the surface of an alveolus and the determination of an alveolus as the mathematical terminus of the tree structure. A $20 \mu \mathrm{m}$ limitation was set for the lower limit of ductal length in our MATLAB based correction of the branching algorithm. This had the practical effect of combining points which were clearly very short branches (a few microns) or clearly small bumps on the complex surfaces of alveoli. These areas resulted from the Amira analysis having disregarded scale. Next, we performed a complete generational analysis shown using color-coding to reflect branch generation.

The corrected tree analysis of the selected region of around $350 \mu \mathrm{m}$ cubed contained 69 ducts and 104 alveoli (Fig. 3c-d), where ducts are color coded by generation and the alveoli are shown as small red circles with a thin dashed line representing their length. The color coding is not the same as in our ductal analysis in Fig. $3 \mathrm{f}-\mathrm{g}$. The analysis indicates a calculated repeating ductal length of $60 \mu \mathrm{m}$ from generation 11 at the lung surface to generation 1, 10 branches more proximal. However ductal diameter decreased from $140 \mu \mathrm{m}$ at generation 1 to $60 \mu \mathrm{m}$ at generation 10 nearest to the pleura.

Finally, we compared the determination of alveolar number above with another method placing spheres of radius $15 \mu \mathrm{m}$ within the volume which touches the curved surface and not overlapping each other. This analysis results in 132 spheres in contact with the surface. The entire volume in Fig. $3 \mathrm{j}$ and magnification in Fig. $3 \mathrm{k}$ showed some double concave shapes, yet the method displayed similar numbers of alveoli.

Alveoli of other mammals. In the spirit of placing our data in a larger contest mentioned previously by others ${ }^{19}$, we investigated connection between lung structural and functional parameters across mammals. We observed a linear correlation between alveolar surface area Vs oxygen consumption, breathe air out Vs breaths/min, alveolar diameter Vs breaths/min, and metabolic rate Vs alveolar diameter across mammalian species of widely differing in body mass. The ratio of time per breath to the diffusion time of oxygen in the alveoli slightly decreases in proportion to alveoli diameter in the mammals (Fig. $4 a-$ e). Breath air out, oxygen consumption, metabolic rate, alveolar diameter and surface area increase while breathing rates decrease as mammals become larger across species $5,20,21$, $22,23,24,25,26,27,28$. 


\section{Discussion}

Surface-to-volume ratio of alveoli. What does a consistent and constant surface to volume ratio imply? We think it implies that the adolescent lung maintains its physiological function for gas exchange while it grows cubically in overall size by increasing alveolar number, while maintaining a constant surface to volume ratio. While this is perhaps not a totally surprising result, the implications of it had not perhaps been fully appreciated by previous structure-function studies of lung development. The increase in overall lung volume and alveolar number thus efficiently matches the increasing metabolic demand for oxygen of the larger organism.

We suggest that the air exchange surfaces likely expand into and adapt to the available space while maintaining a constant surface-to-volume ratio through the addition of a standard size of alveoli, with relatively small adjustments of ductal length and volume. The average size of the alveoli remains nearly the same because the fundamental biophysics of ventilation and perfusion matching changes little as the animal matures. We believe that understanding the process of adaption and optimization likely through matrix changes is critical to understanding how to prevent disease by optimizing gas exchange and envision ways to repair the gas diffusion surface. It is at this level where genetic encoding convolves with biophysical constraints or rules. This interplay yields a variety of shapes of alveoli centered around a robust mean. It is tempting to speculate that the smaller alveolar spaces may represent regions of future expansion into available space. However, our data do support the model that some alveolar ducts will become wider and longer during development. Ducts will then have varying numbers of alveoli subtended onto them, while these alveoli remain the same size independent of duct generation number. Further, it appears likely that some alveoli may transition back into ducts which may give rise to additional alveoli.

Interestingly, ducts more than 10 branches from the pleural surface have almost the same surface-tovolume ratio as alveoli. Thus, this evidence suggests that the ductal network calibrates its diameter based on the volume of air flowing through it, with small changes in diameter and thus small changes in the surface to volume ratio. It has been suggested previously that alveolarization was suggestive of bubble formation ${ }^{29}$ and we support the concept of extrusion of alveolar surfaces into the mesh of the thinning matrix. Recent studies visualizing early alveologenesis in real-time appear to support our ideas about the cubic expansion of the gas diffusion surface throughout adolescence with alveoli springing from specific areas within the matrix ${ }^{11}$. That the surface to volume ratio remains constant through adolescent lung growth allows for the maintenance of lung function optimized for gas exchange with lung volumetric growth.

Murine lung ductal and alveoli analysis. In the fine analysis, where one duct ends or an alveolus begins and ends is quite difficult to determine. It was observed visually that alveoli often have a constriction at their mouth as previously reported ${ }^{30}$, but no analysis of this feature was performed. Our modified analysis indicated the presence of 104 alveoli in the connected component of interest, while our spacefilling analysis indicated room for 88 spheres. Many alveoli seem to have a double concave surface wherein two spheres can be fitted (Fig. 3j - k). The tree analysis identified 16 additional small alveoli. 
While very distal alveoli and ducts have similar sizes, our method allows us to rigorously separate ducts from alveoli in 3D. Specifically, the typical individual alveolar structure has a wide variety of shapes and sizes as can be estimated from the calculated values derived from the Amira tree algorithm in Fig. 1 and as described in Figs. $3 \mathrm{~h}-\mathrm{k}$. The typical alveolus, not the average, is $50 \pm 20 \mu \mathrm{m}$ in diameter by inspection with a slightly flattened structure as observed by the alveolar length at this degree of inflation. Our measured numbers match closely the $58 \mu \mathrm{m}$ average diameter of the ideal spherical alveolus calculated from the $Y$-intercept of the surface to volume ratio. This is $3 / 4$ the diameter we calculated using the classical linear mean intercept method $45 \mu \mathrm{m} \pm 4 \mu \mathrm{m}, \mathrm{N}=3$ slices, which matches with previously published finding ${ }^{5,31}$. Thus, we consider our measurements and those of the literature for alveolar size to be largely in agreement.

Are the observed double concave alveoli the beginning of two? Perhaps this is so, but such shapes may also be seen in the adult. What is clear is that the air exchange surfaces of the lung are often modeled as simple open spheres, but they are geometrically far more complex than that, with similar scales for both small ducts and alveoli. Because we clearly observed capillaries in the ductal walls, we suggest that the distal alveolar area should perhaps be considered as a "Respiron" or gas exchange unit. However, we made no attempt to measure vascular density in the alveoli or ductal surfaces, precluding quantitative comparisons. The constant surface-to-volume ratio of these structures may also reflect optimal alveolar wall structure, where the appropriate density of capillary networks and elastic matrix complete and maintain integrity of the alveolar structure, thus achieving and maintaining an efficient gas exchange during the respiratory cycle and during expansion as the lung grows in volume.

Alveoli have broadly similar shapes and sizes during growth, but their numbers increase linearly with lung volume. We anticipate that the size of the alveoli in a given mammal is ultimately dependent on oxygen demand and oxygen transport capacity, which is related to body size as well as the dwell time of the air and blood within the lung, which determines the efficiency of gas exchange over a given breath. The absolute size of alveoli and ducts are very similar at the distal regions of an acinar sac, regardless of their distance from the BADJ or parent duct generation number. Thus, we suggest that a large number of new alveoli must form quite rapidly and uniformly as the lung volume expands, while the interstitial matrix remodels and thins and adjusts its elasticity and compliance. Likely the gas diffusion surface behaves as a unit, with new alveoli forming and enlarging quickly to match volume increases in different areas of the acinar sac.

Similarly, to what was originally postulated by Galen (AD 129 to c. 216$)^{32} 33,34$ and observed by others ${ }^{8}$, 35,36 , we therefore now propose that alveolar growth during adolescence resembles the growth in a volume of freely expanding foam. The forces exhibited by the drying and hardening surface reflect the forces that are determined by the matrix structure. The surface expands with bulbous regions enlarging until a new bulbous region breaks out of the weakest spot. This process is repeated resulting in continuously connected but smaller bulbous regions distal to the origin, with the short axis perpendicular to the surface of origin. In the biological parallel of the lung, the matrix governs the biophysics of the surface and thus the point of expansion of ducts and their ultimate size. 
Finally, we observed subtle variation in alveolar size, ductal size, and length during adolescence which may be real because two other recent studies also contained hints of slight variation in alveolar size, following the same pattern at the same or similar stages 5,10 . The average alveolar size seems to decrease slightly in young adulthood. Further careful measurements of individual alveoli would be required to tease out these subtle relationships during the bulk phase of alveolarization.

In summary, we suggest that new alveoli develop into the new space of the expanding chest in a relatively fast continuous process linked closely to lung volume increase. Concomitant matrix and vascular remodeling allow for the maturation and optimization of the perfusion ventilation match. These new alveoli are slightly wider than they are long, but most spaces are very close in relative size suggesting a duct maybe lengthening by recruiting one of its alveoli. Thus, a terminal air space may enlarge through expansion of similar-sized ducts and alveoli into the available space. Matrix remodeling is a key component of this model. Our model predicts that a ductal segment may end abruptly when no space is available or keep lengthening if space allows. Globally, the airway exchange region appears less and less similar to the organized generational branching of the conducting airway as we move more distal down the ductal network as previously proposed ${ }^{37}$.

Alveoli of larger mammals. Because the absolute surface to volume ratio in the mouse lung seemed to be an important feature of the mouse lung itself over a 5-fold increase in volume, we decided to try to place this rule in the context of larger mammals. The plot across species of alveolar size versus other factors related to size was linear as is the case for many parameters ${ }^{19}$. The dynamics of oxygen transport into the lung and its diffusion across the membrane into pulmonary capillaries is a multi-step process with the limiting kinetics involved dependent on the physiological state of the lung in terms of resting Vs exercise and health Vs disease. The limiting kinetics have not been exactly defined ${ }^{38}$. Herein, we focus on the diffusion time versus alveolar size, ignoring convective processes which would only decrease the time required for oxygen to reach the alveolar surface. The average length a molecule of oxygen would have to diffuse during a breath is $25 \mu \mathrm{m}$ in a mouse and $6200 \mu \mathrm{m}$ in an elephant. We reasoned that larger diffusion distances would require more time in a non-linear fashion. The time $(t)$ for the diffusion of oxygen across the radius of a sphere is approximately $x^{2} / 2 D$ ( $D$ is the diffusion coefficient of a solute in free solution and $x$ is the mean distance traveled by the diffusing solute in one direction along one axis after elapsed time $t)^{39,40,41,42}$ which would not be linear with alveolar size. We postulated that the breathing rate volume with size might compensate for the longer time, but it also is linear with size and thus does not. We propose therefore that oxygen diffusion is not the determining factor in the alveolar size, or the surface-to-volume ratio observed herein in a murine species and by extension is likely true in other larger mammalian species. The high ratio of diffusion time to alveolar size indicates that diffusion within the alveoli of mammals is highly unlikely to play a role in evolution based on oxygen determining their ultimate diameter. Interestingly the ratio of diffusion time to alveolar size decreases with size so that in elephants the time for diffusion is within an order of magnitude of that required for diffusion to have an effect, whereas in a mouse it is 4 orders of magnitude too fast to have an limiting effect. Accordingly, convective mechanisms are most likely to make a difference in larger mammals. One of us suggests

Page 10/23 
residence time of the blood in the capillaries is the more likely determinant of alveolar size and by extension the absolute ratio of surface area to volume in a mammalian lung. Accurate direct measurements and more accurate models are needed to make a final determination of the limiting factors in oxygen transport across mammalian spices. We propose that studies of surface-to-volume relationships and perfusion ventilation matching within species and between mammalian species will yield important information about the functional properties of the mammalian lung and holds important information about both maintaining a healthy lung and envisioning rebuilding, repairing, or mimicking of normal function.

\section{Methods}

Mice. All animal procedures were performed in strict accordance with the National Institutes of Health $(\mathrm{NIH})$ Guide for Care and Use of Laboratory Animals and approved by the Children's Hospital Los Angeles Animal Care and Use Committee. A 6-8 weeks old male and female homozygous mice for the targeted mutation ROSA-mT/mG on a C57BL/ 6 background (Stock No. 007676) were purchased from Jackson Laboratory (Bar Harbor, ME, USA). ROSA-mT/mG is a ubiquitously expressed, cell membrane-targeted, two-color fluorescent Cre-reporter transgene ${ }^{16}$. In the absence of Cre recombinase, $\mathrm{mTmG}$ mice constitutively express membrane-targeted tdTomato red fluorescent protein. Following exposure to Cre recombinase, the tdTomato expression cassette is excised, and the rearranged $\mathrm{mTmG}$ transgene converts to an expression of a membrane-targeted GFP (enhanced green fluorescent protein). In this study, Cre mediated recombination was not used, and only the ubiquitously expressed membrane targeted tdTomato was imaged. The animals were housed in the animal facility allowed food and water ad libitum, and breeding was carried out to produce homozygote ROSA-mT/mG mice. The postnatal mice of age groups $7,14,21$, and 28 days were used for further studies.

Inflated lung preparation. Inflated lungs were prepared from homozygous ROSA-mT/mG mice of all 4 different age groups. Anesthesia was induced via intraperitoneal injection of ketamine $100 \mathrm{mg} / \mathrm{kg}$ (Henry Schein Inc.) and xylazine $10 \mathrm{mg} / \mathrm{kg}$ (Akron Inc.) solution mixture. Once anesthetized, a cervical tracheotomy was performed and a $24 \mathrm{G}$ angiocatheter was positioned in the distal trachea and secured with a 3-0 silk tie. The mouse was then ventilated with the VentElite rodent ventilator (Harvard Apparatus) at 120 breaths/min, volume $400-500 \mu \mathrm{L}$, peak inspiratory pressure $21.5 \mathrm{~cm} \mathrm{H}_{2} \mathrm{O}$, and positive end-expiratory pressure $3 \mathrm{~cm} \mathrm{H}_{2} \mathrm{O}$.

Perfusion and fixation solutions were degassed under a vacuum for several hours before use. Access to the vasculature was achieved by surgical exposure of the abdominal viscera and insertion of a $24 \mathrm{G}$ safety intravenous catheter into the inferior vena cava. The descending aorta was severed, and blood was flushed from the vasculature with $100 \mathrm{ml}$ saline solution (Baxter) containing 6U heparin (Sigma Aldrich) at $24 \mathrm{~cm} \mathrm{H}_{2} \mathrm{O}$. The breathing cycle was maintained during the flush. Once the perfusate ran clear, the breathing cycle was stopped, and tidal pressure was held at $20 \mathrm{~cm} \mathrm{H}_{2} \mathrm{O}$. Fixation was performed by perfusing $50-100 \mathrm{ml}$ of a freshly prepared fixative solution containing $3 \%$ formaldehyde (Sigma Aldrich) 
prepared in 1X phosphate-buffered saline (PBS). After perfusion with fixative, the trachea was tied off with a silk tie to maintain lung inflation. The fixed, inflated lung with the attached heart was carefully removed from the chest cavity and submerged in the post-fix solution containing $2 \% \mathrm{MeOH}-$ free formaldehyde (Polysciences Inc.) prepared in $1 \mathrm{X}$ PBS and allowed to post-fix for two days at $4^{\circ} \mathrm{C}$. The fixative was removed by washing with twice daily buffer changes with 1 X PBS containing $0.02 \% \mathrm{NaN}_{3}$ (Sigma Aldrich) for 4 days at $4^{\circ} \mathrm{C}$. Then, the still-inflated lungs were transferred into a permeabilization solution mixture containing 1 X PBS, 1.0\% w/v Triton-X100 (Sigma Aldrich), and 0.02\% w/v NaN3 (Sigma Aldrich) and stored submerged for several months at $4{ }^{\circ} \mathrm{C}$. The permeabilization solution was replaced with a fresh solution every month. As the lungs permeabilize, trapped air inside the lung tissue slowly escapes and is replaced by the buffer solution until the lung is no longer buoyant and sinks.

After samples were sufficiently permeabilized, the Triton-X100 was washed out by soaking the sample in a solution mixture of $1 \mathrm{X}$ PBS and $0.02 \% \mathrm{w} / \mathrm{v} \mathrm{NaN} \mathrm{N}_{3}$ with daily buffer changes over a week at $4^{\circ} \mathrm{C}$. The lungs were then dissected into their constituent lobes and stored at $4^{\circ} \mathrm{C}$ in a solution mixture of $1 \mathrm{X}$ PBS and $0.02 \% \mathrm{w} / \mathrm{v} \mathrm{NaN}$. Mouse lungs prepared in this manner maintain their inflated morphology and exhibit robust fluorescent protein fluorescence for at least one-year storage.

Sample preparation for tomographic imaging. All infiltration and polymerization procedures were performed at room temperature and protected from light whenever possible to prevent photobleaching of the fluorescent proteins. Permeabilized lung samples were infiltrated by stepping through a step series of increasing concentrations of acrylamide and bis-acrylamide solution, 37.5:1 (BioRad) prepared in a 1X PBS solution containing TEMED [0.6 $\mathrm{L} / \mathrm{ml}]$ (BioRad). Acrylamide infiltration solutions were prepared fresh and degassed under vacuum for a minimum of 3 hours at room temperature before being added to the samples. Infiltrations were performed at room temperature in plastic 6 well dishes on a platform shaker with gentle shaking. Samples were equilibrated in each acrylamide concentration sequentially, $1 \mathrm{X}$ $4 \%, 1 X 8 \%$, and $2 \times 12 \% \mathrm{v} / \mathrm{v}$, for $24 \mathrm{~h}$ before being transferred to a new well containing the next acrylamide solution. After infiltration, an embedding-polymerization solution consisting of $12 \% \mathrm{w} / \mathrm{v}$ acrylamide-PBS was prepared in $15 \mathrm{ml}$ batches and degassed under vacuum for a minimum of 4 hours. Immediately before embedding, $70 \mu \mathrm{L}$ of freshly prepared $10 \% \mathrm{w} / \mathrm{v}$ ammonium persulfate (BioRad) solution was added to the acrylamide solution and gently mixed to avoid excessive in-folding of air.

For casting the acrylamide, wells of glass spot plates (Pyrex) were filled with the embedding-polymerization solution and the lung samples were immediately transferred into the well and oriented. Note that gravity will draw the sample to the bottom of the well, and that will later be the top of the sample for imaging. The spot plate wells were then capped with $25 \mathrm{~mm}$ round cover glasses to seal out air which would impede polymerization. Absorbent paper towel tips were used to draw away excess solution and enhance the airtight seal of the cover glass onto the well opening. The remaining embedding-polymerization solution was placed aside in a sealed tube and served as an indicator of complete acrylamide polymerization. Once the acrylamide was fully polymerized, usually $2-3 \mathrm{~h}$, the cover glass cap was lifted and the acrylamide hemisphere containing the embedded lung sample was liberated 
from the glass well and transferred to a solution of 1X PBS containing $0.02 \% \mathrm{w} / \mathrm{v}$ NaN3 (Sigma Aldrich) for storage.

Tomographic Imaging. Serial confocal tomographic imaging (referred to as Vibratome Assisted SubSurface Imaging Microscopy, Vibra-SSIM) was performed by coupling an upright confocal microscope to a modified vibrating microtome tissue sectioning device mounted adjacent to the microscope stage. The sectioning device assemblage consisted of the vibrating head, blade assembly, and knife holder from a salvaged Vibroslice Vibrating Microtome (Model \#NVSLM, World Precision Instruments) that was mounted onto an XYZ stage (Newport Instruments) equipped with manual vernier micrometer actuators (Newport) on the $X$ and $Y$ axes for alignment. A CONEX-LTA-HS DC servo motorized actuator (Newport) controlled via CONEX-CC Controller software (Newport) was attached to the Z-axis and was used to control knife height. The sectioning device assemblage was mounted onto a $90^{\circ}$ platform (Newport) and placed onto a damper rod with a mounting base (Newport). The entire sectioning device and postassembly were bolted to the air table adjacent to the microscope stage. The knife vibration frequency was driven by voltage from a generic DC power supply. Single edge blades were purchased from Lafayette Instrument Co (Model 752/1/SS).

Before imaging, a sample embedded in an acrylamide hemisphere was glued with cyanoacrylate adhesive (Loctite 404) with its flat side down onto the bottom of a homemade acrylic tray. This try will serve as the sample bath. The sample bath was mounted to the motorized microscope stage. The sample bath was filled to above the top of the acrylamide embedded sample with room temperature 1X PBS that had been degassed under vacuum overnight. Imaging was performed using a Zeiss LSM710 confocal microscope on an Examiner upright stand and equipped with a fixed height motorized microscope stage (Prior Scientific). Zen Black software (Zeiss) was used to control the XY tiled, Z stack imaging. In addition to $X Y$ tiling, the motorized stage is also used to move the sample from the imaging location to the knife location for the removal of the sample thereby providing access to the next underlying region of the sample. The imaging session starts 10 microns below the upper surface of the acrylamide block. This ensured that the surface of the acrylamide block was never included in the resultant $Z$ stack layer and any knife cutting induced distortions were avoided. The imaging-cutting sequence was standardized as follows: 60 microns $Z$ stacks tiled in $X$ and $Y$ were acquired with the microscope. Then, the vibrating microtome was used to shave off the top 40 microns from the acrylamide block. Then, the microscope focus was lowered by 40 microns and another series of tiled 60 microns $Z$ stacks was acquired. Then, the knife was lowered by 40 microns and another layer of the block was removed. Then the microscope focus was lowered by 40 microns and another series of tiled 60 microns $Z$ stacks was acquired. The sequence was repeated until the desired extended $Z$ regions were covered. This reiterative process generates consecutive, $X Y$ tiled, $Z$ stack layers that overlap in $Z$ with their next underlying $X Y$ tiled, $Z$ stack layer.

All imaging was performed using a Zeiss 20x 1.0NA Plan-Apochromat water dipping objective. A $543 \mathrm{~nm}$ HeNe laser was used to excite tdTomato fluorescent protein and the emission was collected between 550-740 nm. Imaging was performed with Zen Black software (Zeiss). Images were collected in 16-bit 
depth. Tiled $Z$ stacks were collected in $2 \times 2$ rectangular grids with $10 \%$ overlap between adjacent tiles. Auto $Z$ brightness correction was used to mitigate depth-related decreases in signal intensity. A pinhole of 0.85 Airy units was used to collect 1.5 micron thick optical sections and the inter-section interval was 0.74 microns. Resultant Z stacks were saved in Zeiss CZI file format.

Post processing and extended $Z$ stack compilation. The $X Y$ tiled $Z$ stack layers were stitched together using either Zen Black or Zen Blue software (Zeiss). To compile extended image volumes, sequential composite image $Z$ stacks were compared visually in the region of $Z$ overlap between the two. Based on best correspondence in the $Z$ overlap region, the bottom of the upper composite $Z$ stack and top of the underlying stack was selected. Once the junctions in $Z$ were determined for all of the sequential composite $Z$ stacks, the optical sections were exported as 16-bit TIFF files and compiled into a larger stack of images that encompassed the entire extended volume. The sequential $Z$ stacks often exhibited slight offsets in $X$ and $Y$, most likely due to mild inaccuracies in the motorized stage movements. To correct this, the compiled larger Z stack was imported into FIJI software ${ }^{43}$ and processed with the Register Virtual Stack Slices ${ }^{44}$ plugin using the translation-only registration model with the shrinkage constraint option. After registration of the extended Z stacks, they were imported into Imaris 8.4.2 (Biltplane) or Amira 19.2 software for further analysis.

Image processing of confocal microscopy raw data. From the confocal microscopy raw data, the airspaces were extracted by first binarizing the tissue. The 16-bit images were binarized by using the Chan-Vese (CV) algorithm, a level set-based method ${ }^{17}$. A program was implemented in $\mathrm{C}++$ using the semi-implicit Euler method, applying the Additive Operator Scheme to decrease calculation time $\mathrm{e}^{45,46}$. The parameters in the CV binarization were $\varepsilon=5 / \mathrm{pi}, \mu=0.00725125, \lambda=200,000$, and time step $\Delta t=2.0$. These parameters were determined empirically by overlaying the output of the binarization procedure with the raw data in Imaris 8.4.2 software and validating the results, adjusting the parameters as required. The binarized volume was an output a stack of 16-bit TIFF images with each voxel of a value 65,535 or 0 representing tissue or airspace voxels, respectively.

The remaining steps were performed using MATLAB software. Since the binarization captured some of the noise presents in the volume, a 3D isotropic Gaussian Filter of standard deviation 3 was applied to the volume. After the Gaussian Filter was performed, the image was no longer binary, then a threshold was applied so that voxels below a certain threshold were set to 65,535 and the rest to 0 .

Morphological closing was then performed on the images to remove connections between individual acini due to features of the lung such as Pores of Kohn and imaging artifacts such as light intensity variations. A 2-D disk of radius 11 was used as a structuring element on each image individually. Finally, the airspaces were extracted by running a connected component algorithm that grouped voxels so that voxels that shared a face (3-D 6-connectivity) belonged to the same set. Each connected component was then output as a separate stack of 16-bit TIFF images. 
Spots creation in Imaris software to statistically analyze numbers and position of alveoli. The data from MATLAB pipeline was uploaded into Imaris 8.4.2 software. A connected component data volume was then created. A semi-transparent surface was created for the airspaces in the volume which show the alveoli. The volume rendering itself was then turned off to allow only the surfaces to be visible. After surfaces were created the spots feature was used to create easily visible spheres. The setting for spots depends on the size of the spaces in which we want to place the spots. The size that correctly positions one spot into each alveolus is diameter $X, Y$, and $Z=15 \mu \mathrm{m}$, Area $=225 \mu \mathrm{m}^{2}$. This setting is specific to the consistently observed volume of the space in each alveolus throughout the connected components examined in this research. The spots (spheres) were then assigned colors that are visible in the alveoli. Then, alveoli sitting on cut surfaces of the connected components and therefore not completed were then easily visible and deleted to avoid measurement distortion. Imaris then counted and analyzed the number of alveoli as needed through statistical operations that are built-in Imaris. While rendering more than one connected component at a time, each component's semi-transparent surface was given a contrasting color to its adjacent or if necessary, each set of spots in each component was also color adjusted for visual clarity (Fig. 3 j - k).

Centerline Tree Determination in Amira. The data set was read into Amira 19.2 software and the centerline tree algorithm was applied unchanged. The algorithm was allowed to pick the root: setting -1 . The slope was varied between 0.5 and 5 as was the zero-value. The resulting centerline trees were visualized within the original semi-transparent volume. A specific value of these two parameters had the effect of clearly missing some small branches and end-nodes (alveoli) but exhibiting the correct scale to picking up most of the alveoli. Other values have an improper scale which results in fine surface details being labeled branch nodes with an excessive number of end-nodes.

This property was useful for visualization because one could prune smaller branches and alveoli as shown in Fig. $3 b$ (slope $=3$ and zero-value $=4$ ). The value of the parameters to create the best balance between selecting every surface bump to be alveoli and missing smaller branches and end-nodes was determined to be a slope of 1.5 and a zero-value of 4 is shown in Figs. $3 c-d$. This set of parameters missed very few alveoli but over-determined their number and placed branching nodes close together. Hence the refinement is described below.

Generation Analysis of Lung Tree. The co-ordinates representing nodes and segments of the lung tree were obtained from Amira 19.2 software. These data served as input data to construct lung tree generation in MATLAB software. The lung tree originates from the root node is shown in red color in Fig. 3e. The MATLAB performed a merging operation based on the Euclidian distances and connections between nodes to eliminate the redundancy produced by Amira software. The root of the tree was then identified by the thickness analysis along all branches. From the root node, a growing process was adopted to reclusively check all branches to decide the generation number. All the nodes with no further connections are considered as the end nodes. These end nodes may partly indicate the position of alveoli. 


\section{References}

1. Takebe T, Wells JM. Organoids by design. Science 364, 956-959 (2019).

2. Dye BR, et al. In vitro generation of human pluripotent stem cell derived lung organoids. Elife 4, (2015).

3. Lucas A, Yasa J, Lucas M. Regeneration and repair in the healing lung. Clin Trans/ Immunology 9, e1152 (2020).

4. Hogan BL, et al. Repair and regeneration of the respiratory system: complexity, plasticity, and mechanisms of lung stem cell function. Cell Stem Cell 15, 123-138 (2014).

5. Pozarska A, et al. Stereological monitoring of mouse lung alveolarization from the early postnatal period to adulthood. Am J Physiol Lung Cell Mol Physio/ 312, L882-L895 (2017).

6. Maynard RL, Pearce S, Nemery B, Wagner PD, Cooper B, Cotes JE. Cotes' lung function, Seventh edition. edn. Wiley-Blackwell (2020).

7. Melen E, Guerra S. Recent advances in understanding lung function development. F1000Res 6, 726 (2017).

8. Schittny JC. Development of the lung. Cell Tissue Res 367, 427-444 (2017).

9. Metzger RJ, Klein OD, Martin GR, Krasnow MA. The branching programme of mouse lung development. Nature 453, 745-750 (2008).

10. Schulte H, Muhlfeld C, Brandenberger C. Age-Related Structural and Functional Changes in the Mouse Lung. Front Physiol 10, 1466 (2019).

11. Hsia CC, Hyde DM, Weibel ER. Lung Structure and the Intrinsic Challenges of Gas Exchange. Compr Physiol 6, 827-895 (2016).

12. Crapo JD, Barry BE, Gehr P, Bachofen M, Weibel ER. Cell number and cell characteristics of the normal human lung. Am Rev Respir Dis 126, 332-337 (1982).

13. Hsia CC, Hyde DM, Ochs M, Weibel ER, Structure AEJTFoQAoL. An official research policy statement of the American Thoracic Society/European Respiratory Society: standards for quantitative assessment of lung structure. Am J Respir Crit Care Med 181, 394-418 (2010).

14. Kitaoka H, Nieman GF, Fujino Y, Carney D, DiRocco J, Kawase I. A 4-dimensional model of the alveolar structure. J Physiol Sci 57, 175-185 (2007).

15. Weibel ER. Lung morphometry: the link between structure and function. Cell Tissue Res 367, 413426 (2017).

16. Muzumdar MD, Tasic B, Miyamichi K, Li L, Luo L. A global double-fluorescent Cre reporter mouse. Genesis 45, 593-605 (2007).

17. Chan TF, Vese LA. Active contours without edges. IEEE Trans Image Process 10, 266-277 (2001).

18. Mund SI, Stampanoni M, Schittny JC. Developmental alveolarization of the mouse lung. Dev Dyn 237, 2108-2116 (2008). 
19. Lindstedt SL. Body size, time and dimensions of oxygen diffusion. Comp Biochem Physiol A Mol Integr Physiol 252, 110847 (2021).

20. Parent RA. Comparative biology of the normal lung, Second edition. edn. Elsevier/AP, Academic Press is an imprint of Elsevier (2015).

21. Glazier JB, Hughes JM, Maloney JE, West JB. Vertical gradient of alveolar size in lungs of dogs frozen intact. J Appl Physio/ 23, 694-705 (1967).

22. Hyde DM, et al. Alveoli increase in number but not size from birth to adulthood in rhesus monkeys. Am J Physiol Lung Cell Mol Physiol 293, L570-579 (2007).

23. Hyde DM, Tyler NK, Putney LF, Singh P, Gundersen HJ. Total number and mean size of alveoli in mammalian lung estimated using fractionator sampling and unbiased estimates of the Euler characteristic of alveolar openings. Anat Rec A Discov Mol Cell Evol Biol 277, 216-226 (2004).

24. Ochs $M$, et al. The number of alveoli in the human lung. Am J Respir Crit Care Med 169, 120-124 (2004).

25. Siegwart B, Gehr P, Gil J, Weibel ER. Morphometric estimation of pulmonary diffusion capacity. IV. The normal dog lung. Respir Physiol 13, 141-159 (1971).

26. Asgharian B, et al. Development of a rhesus monkey lung geometry model and application to particle deposition in comparison to humans. Inhal Toxicol 24, 869-899 (2012).

27. Brown RE, Butler JP, Godleski JJ, Loring SH. The elephant's respiratory system: adaptations to gravitational stress. Respir Physiol 109, 177-194 (1997).

28. Clark AR, Burrowes KS, Tawhai MH. Ventilation/Perfusion Matching: Of Myths, Mice, and Men. Physiology (Bethesda) 34, 419-429 (2019).

29. Scarpelli EM, Mautone AJ, DeFouw DO, Clutario BC. Intraalveolar bubbles and bubble films: II. Formation in vivo through adulthood. Anat $\operatorname{Rec} 246,245-270$ (1996).

30. Akram KM, et al. Live imaging of alveologenesis in precision-cut lung slices reveals dynamic epithelial cell behaviour. Nat Commun 10, 1178 (2019).

31. Soutiere SE, Tankersley CG, Mitzner W. Differences in alveolar size in inbred mouse strains. Respir Physiol Neurobio/ 140, 283-291 (2004).

32. Fitting JW. From breathing to respiration. Respiration 89, 82-87 (2015).

33. Baker AB. Artificial respiration, the history of an idea. Med Hist 15, 336-351 (1971).

34. Galen, Nicolaus. De usu partium corporis humani, libri XVII. Apud Gulielmum Rovillium (1550).

35. Dishop MK. Diagnostic Pathology of Diffuse Lung Disease in Children. Pediatr Allergy Immunol Pulmonol 23, 69-85 (2010).

36. Nerger BA, Nelson CM. 3D culture models for studying branching morphogenesis in the mammary gland and mammalian lung. Biomaterials 198, 135-145 (2019).

37. Vasilescu DM, et al. Assessment of morphometry of pulmonary acini in mouse lungs by nondestructive imaging using multiscale microcomputed tomography. Proc Natl Acad Sci U S A 109, 17105-17110 (2012). 
38. Martin S, Maury B. Modeling of the oxygen transfer in the respiratory process. ESAIM: Mathematical Modelling and Numerical Analysis 47, 935-960 (2013).

39. Atkins PW. Physical chemistry, 5th edn. Oxford University Press (1994).

40. Lindstedt SL. Pulmonary transit time and diffusing capacity in mammals. Am J Physio/ 246, R384388 (1984).

41. Lambertsen CJ, Clark JK, et al. The pulmonary oxygen diffusion coefficient. Am J Med Sci 218, 715 (1949).

42. Frank AO, Chuong CJ, Johnson RL. A finite-element model of oxygen diffusion in the pulmonary capillaries. J Appl Physiol (1985) 82, 2036-2044 (1997).

43. Schindelin J, et al. Fiji: an open-source platform for biological-image analysis. Nat Methods $9,676-$ 682 (2012).

44. Arganda-Carreras I, Sorzano COS, Marabini R, Carazo JM, Ortiz-de-Solorzano C, Kybic J. Consistent and Elastic Registration of Histological Sections Using Vector-Spline Regularization.). Springer Berlin Heidelberg (2006).

45. Rada L, Chen K. Improved Selective Segmentation Model Using One Level-Set. Journal of Algorithms \& Computational Technology 7, 509-540 (2013).

46. Zahir M, Hemza B, Arezki Y. Semi-Implicit Additive Operator Splitting Scheme for Image Segmentation Using the Chan-Vese model. Sensors and Transducers 215, 35-42 (2017).

\section{Figures}



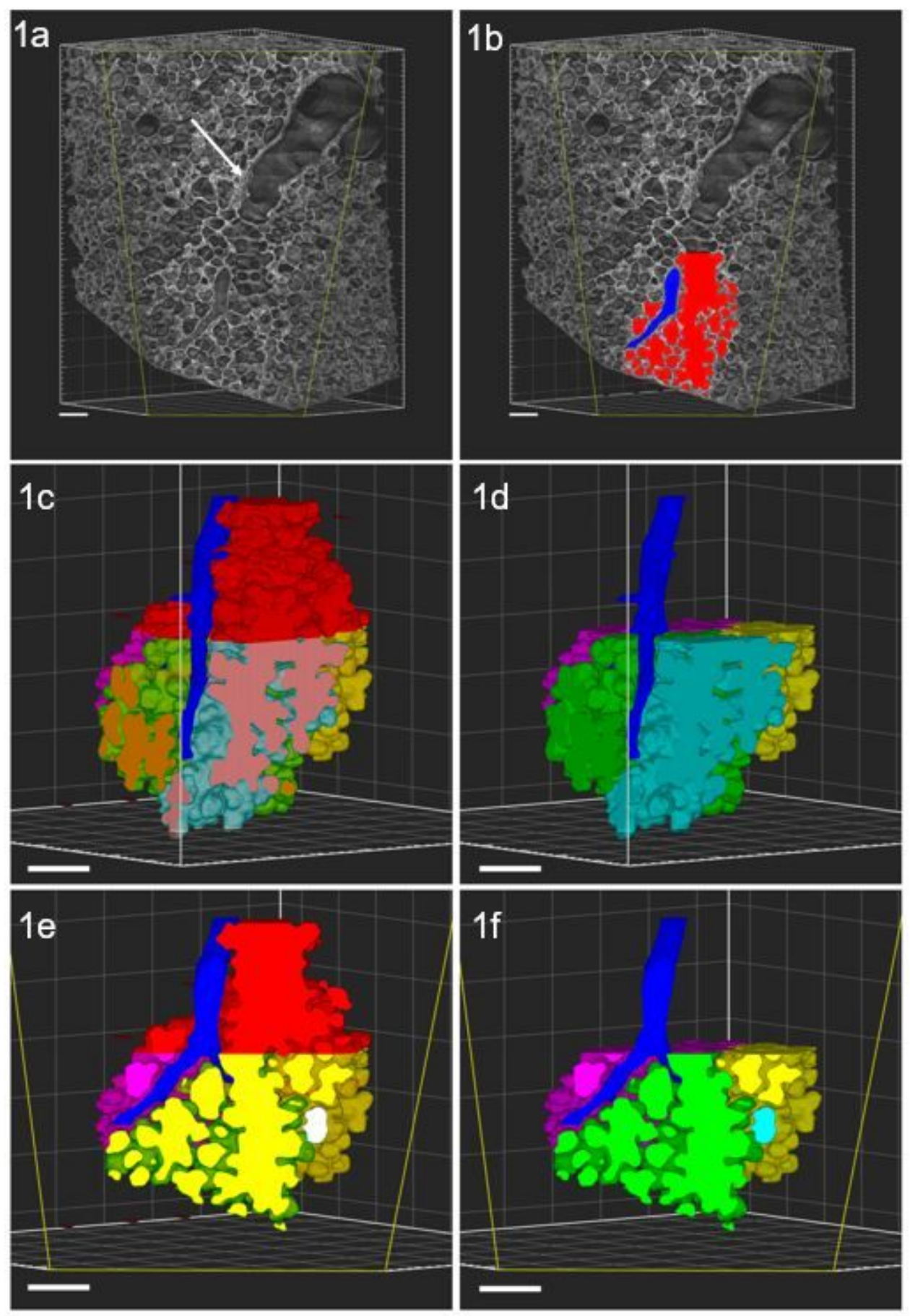

Fig. 1

\section{Figure 1}

Visualization of data set used for segmented surface analysis. 3D image data $(0.42 \mu \mathrm{m} X, Y$ and $0.74 \mu \mathrm{m}$ $Z$ voxel size) in $857 \times 866 \times 1333 \mu \mathrm{m} 3$ image volume showing the selected segmented regions in red, pink, blue, green and violet colors on which surface to volumes measurement were performed. BADJ (white arrow) represent the transition zone. Alveolar ductal branches can be seen leading to the subsaccule regions analyzed. A pulmonary artery in blue is depicted for perspective and aligns parallel to 
alveolar ducts. The figures $1 \mathrm{a}-\mathrm{f}$ is on the same scale bar of $100 \mu \mathrm{m}$ where top portion is proximal towards the lungs while bottom is directed towards pleura.

$2 a$

Surface area to volume ratios Vs Age

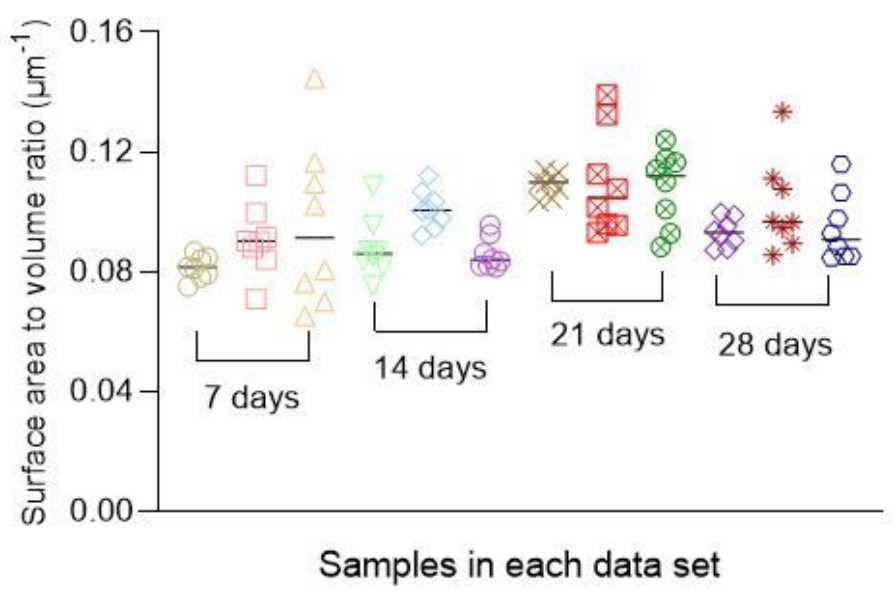

2b Surface area to volume ratios as a function of volume

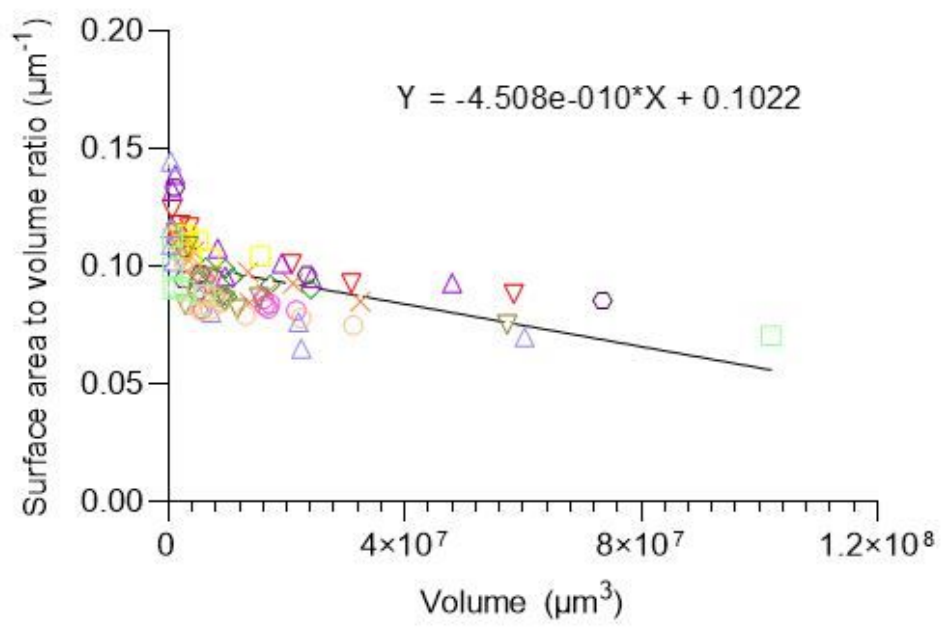

Fig. 2

\section{Figure 2}

Surface to volume ratios of the segmented sub-saccular regions defined by their continuous connectivity (connected components) in postnatal 7, 14, 21 and 28 days old mice (2a); A plot of all the surface to volume ratios as a function of volume showing only a slight decrease in slope with increasing connected components volume (2b). 

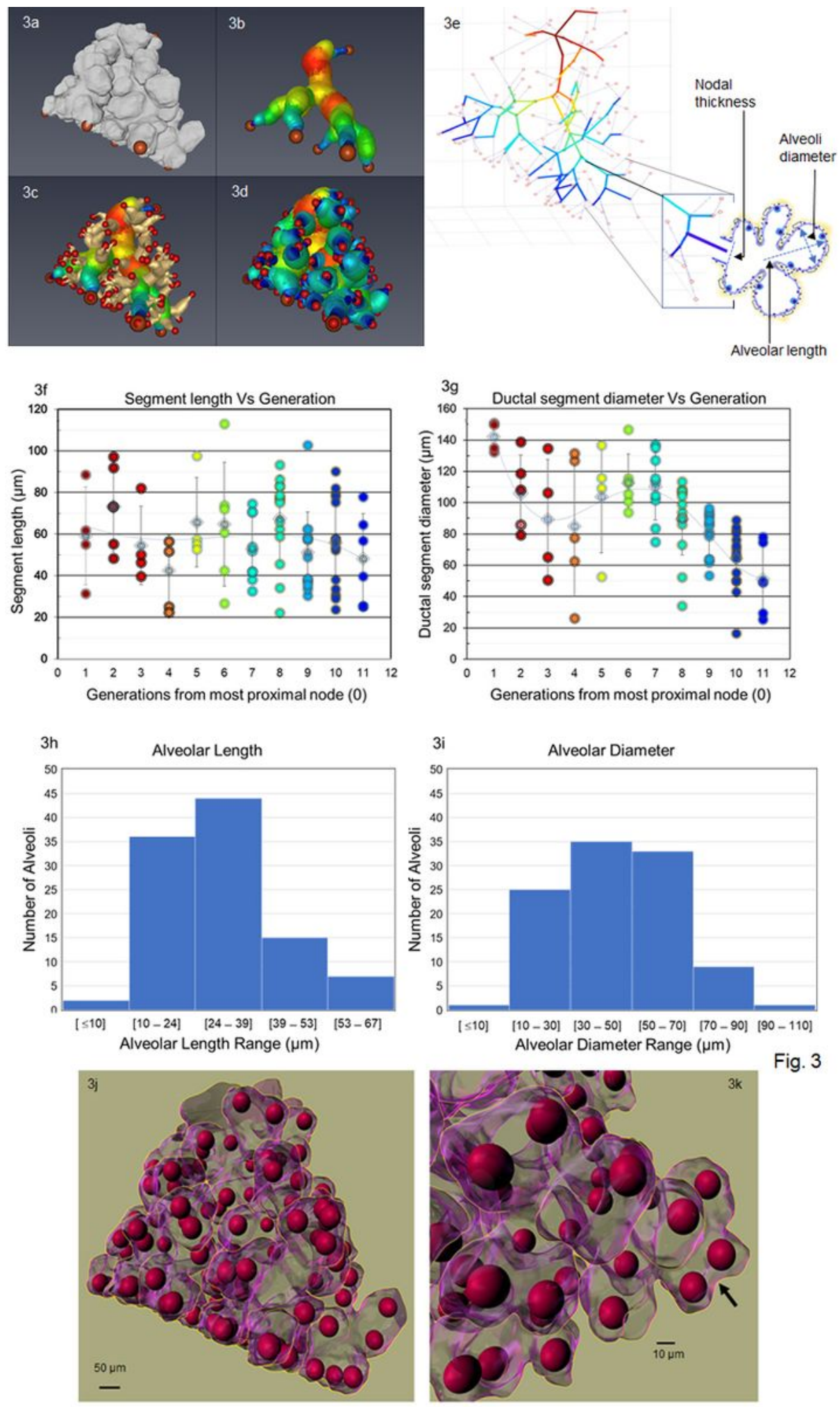

Fig. 3

\section{Figure 3}

Lung tree and branch analysis: Tree branching algorithm of lungs in Amira 19.2 software shows the surface data of the connected components; colors differences represent ductal diameter difference (3a $\mathrm{d}$ not to scale). Generational analysis shows solid lines ductal segments; ductal colors from dark red to light blue represent generation number (3e). The variation in segmental length and ductal diameter from most proximal node in each generation shows sinusoidal pattern $(3 e-g)$. Typical alveoli exhibit $30-70$ 
$\mu \mathrm{m}$ diameter and $24-39 \mu \mathrm{m}$ length $(3 \mathrm{~h}-\mathrm{I})$. Individual alveoli in the connected component on a $50 \mu \mathrm{m}$ scale and alveoli on $10 \mu \mathrm{m}$ scale are shown in $3 \mathrm{j}$ and $\mathrm{k}$, respectively. Arrow in figure $3 \mathrm{k}$ represents double concaved surface (The image of alveoli in Fig. 3e is obtained from doi: 10.1186/s12931-018-0837-5).
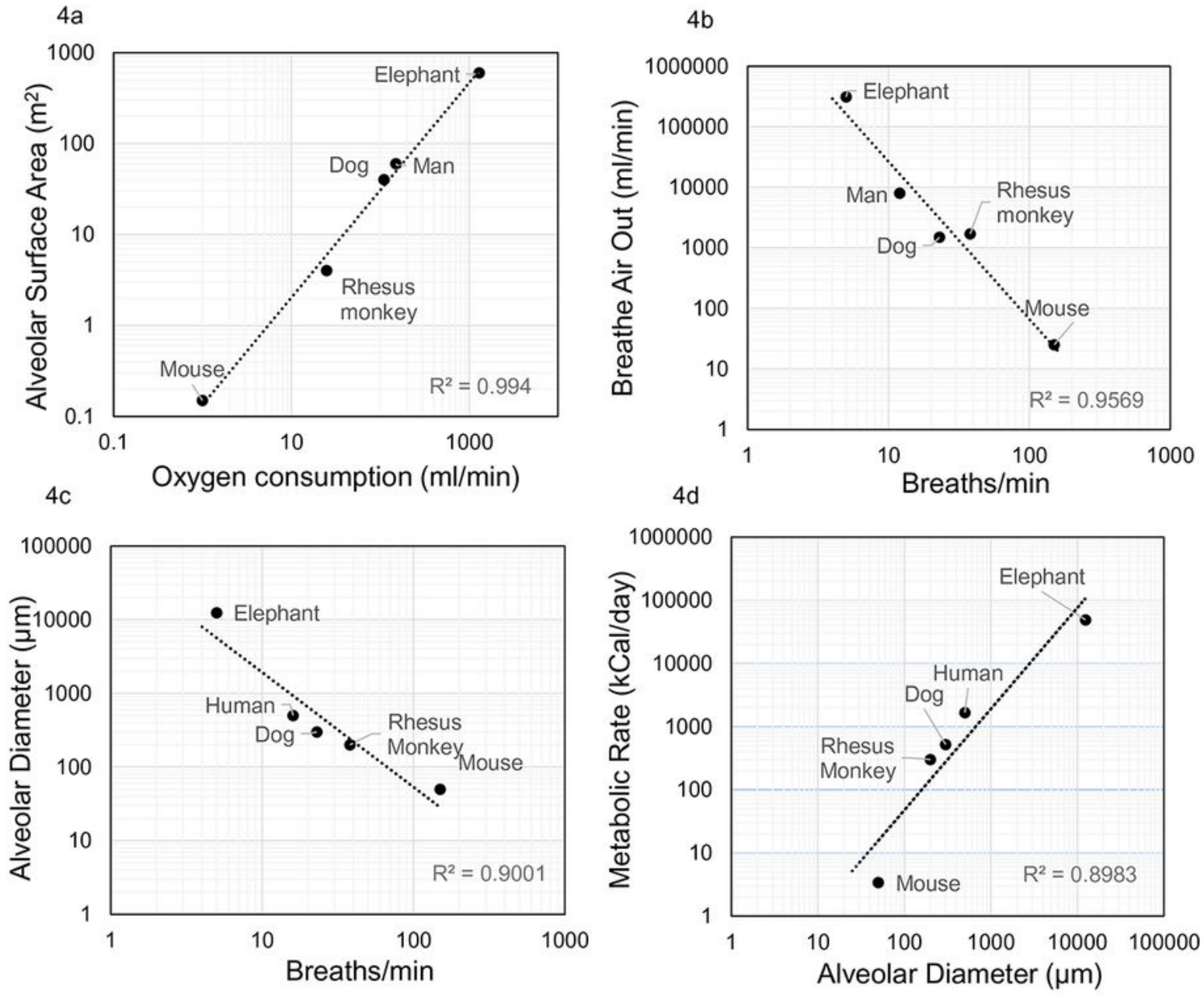

$4 \mathrm{e}$

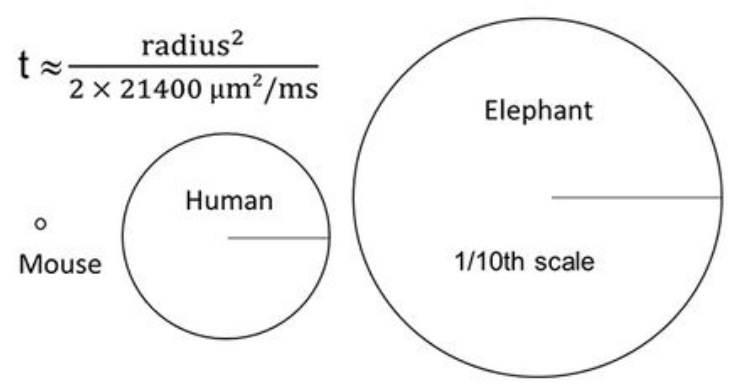

Fig. 4

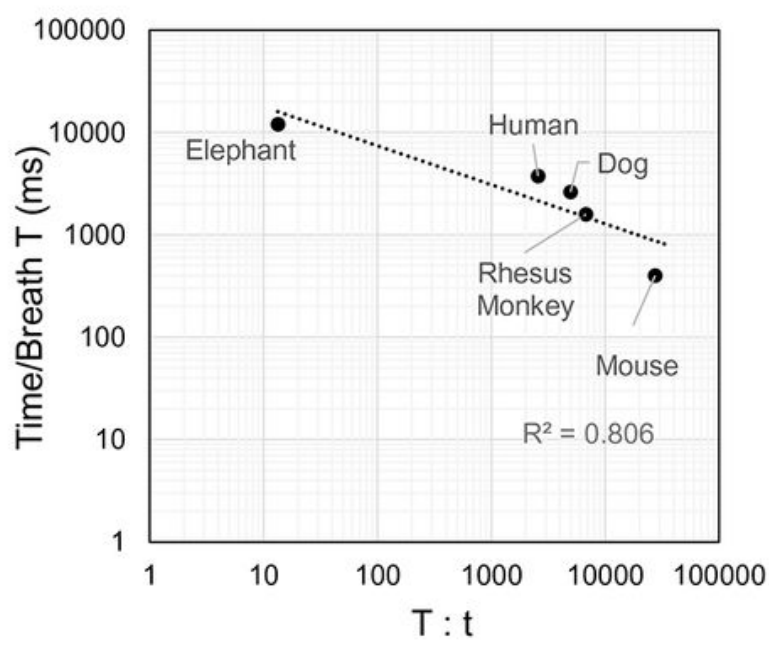

\section{Figure 4}

Correlations observed between alveolar surface area and other parameters: A linear correlation was observed between alveolar surface area Vs oxygen consumption (4a), breathe air out Vs breaths/min 
(4b), alveolar diameter Vs breaths/min (4c), and metabolic rate Vs alveolar diameter (4d) across mammals. The ratio of time per breath to the diffusion time of oxygen in the alveoli is slightly decreasing when in proportion to alveoli diameter in the mammals (4e). The parameter values to plot these graphs were obtained from the references. 